\title{
2006-133: MECHANICAL MEASUREMENTS: REWRITING THE SCRIPT
}

\section{Richard Layton, Rose-Hulman Institute of Technology}

Richard A. Layton earned his doctorate from the University of Washington in 1995 and is currently an Associate Professor of Mechanical Engineering at Rose-Hulman Institute of Technology. Prior to his academic career, Dr. Layton worked for twelve years in consulting engineering, culminating as a group head and a project manager. His professional interests include physical systems theory for modeling and simulation of dynamic systems and curriculum development and lab development in mechanical engineering.

\section{James Mayhew, Rose-Hulman Institute of Technology}

James E. Mayhew received his Ph.D. from the University of California, Davis in 1999. He is currently an Associate Professor of Mechanical Engineering at Rose-Hulman Institute of Technology. Prior to this, Dr. Mayhew was a US Air Force Officer, working in research, development, and logistics engineering for 6 years and teaching aeronautics at the US Air Force Academy for 5 years. 


\title{
Mechanical Measurements: Rewriting the Script
}

\begin{abstract}
In a recent paper, we describe specific steps to improve a mechanical engineering lab sequence by paying close attention to the learning objectives appropriate to engineering laboratories. Several recommendations are made for long-term improvements to our junior-level course in measurement systems. In the near-term, however, we have made substantive improvements to the mechanical measurements course within existing budgets and space and without increasing the course credit hours. These near-term improvements are the topic of this paper. The first improvement is the transformation of measurement-device lectures into 50-minute, "hands-on" mini-labs. The second improvement is the replacement of some labs with workshops on uncertainty analysis and experiment design. The third improvement is the narrowing of the focus of the student projects to allow time for greater depth and to better meet our learning objectives. The fourth improvement is "rewriting the script" of conventional experiments to improve student engagement and reduce the tendency of students to "take the data and get out." We observed improvements in both the quality of the questions students ask during the term and the quality of their final presentations.
\end{abstract}

\section{Introduction}

Traditional design of mechanical engineering laboratories is that of lectures supported by laboratory assignments or vice versa. Typical topics include the principles of measurement devices, data analysis, validation of engineering principles, and some experimental design. The typical objectives of such courses are for students to gain familiarity with basic experimental methods and technology and to validate engineering principles encountered in lecture courses such as system dynamics or fluid mechanics.

From conversations over several years with colleagues from various institutions, we note that mechanical engineering (ME) laboratory courses like ours often suffer from neglect and a low level of student engagement. In a previous paper [1] we describe a plan to improve ME labs by improving student engagement and by more closely meeting the learning objectives appropriate to engineering laboratories. The purpose of this paper is to present our accomplishments to date in the measurements course.

\section{Prior condition of the measurements course}

Our version of Mechanical Measurements is a two credit-hour, junior-level course that meets for one lecture hour and three lab hours per week. Course enrollment is limited to 24 students per section with three sections offered per term. All sections are team-taught by three faculty members. The lecture component of the course covers measurement technology and uncertainty analysis. Prior to our recent modifications, lectures were delivered simultaneously to all three sections in a theater-style lecture hall; students, in teams of four, conducted six scripted experiments and performed a modest experimental project. 
The first main shortcoming of the course in this prior condition was a failure to keep students engaged. As aptly described by a colleague, students tend to adopt a mode of "collect the data and get out" instead of engaging in critical thought [2]. The second main shortcoming was our failure to examine our learning objectives. The course had remained substantially unchanged for at least 20 years.

To address these shortcomings, we used a recently published list of learning objectives for engineering laboratories [3] plus comments from our department's Industrial Advisory Board (IAB) as a starting point for developing our own list of lab objectives. Our list of learning objectives is summarized as follows: "By completing the laboratories in our mechanical engineering curriculum, the student will be able to...

1. apply appropriate sensors, instrumentation, and software tools to measure physical quantities." (Instrumentation)

2. devise an experiment, specify equipment and procedures, implement the procedure, and interpret the resulting data to characterize an engineering material, component, or system." (Experiment)

3. collect, analyze and interpret data, form and support conclusions, make order-ofmagnitude judgments, and know measurement unit systems and conversions." (Data Analysis)

4. recognize unsuccessful outcomes due to faulty equipment, parts, code, construction, process or design, and develop effective solutions." (Learn from Failure)

The first three objectives are met in the measurements course. The fourth objective is met in a senior-level lab course. (Our process and results are described in [1].)

Based on this work, we developed a long-term strategy for transforming and updating the measurements course. Obstacles to implementation are space, equipment funding, and credit hours. In the near-term however, our close attention to lab learning objectives, lab content of prerequisite courses and Advisory Board suggestions has informed our course planning, leading to several substantial improvements to the existing measurements course. Our implementation of these recent course changes is the subject of this paper. Since these changes have been accomplished within existing budgets and space and without increasing the course credit hours, our methods and results should be of interest to ME faculty teaching measurements (and other lab) courses at their institutions.

\section{Overview of near-term improvements}

The recent improvements to the measurements course constitute a dramatic change from our traditional organization and definition of lectures and labs. Lectures have been turned into labs, labs have been replaced with workshops, the experimental project has been carefully constrained, and conventional well-scripted experiments have had their scripts rewritten. Collectively, these changes constitute "rewriting the script" of the traditional course .

Prior to the changes we describe, our class consisted of one fifty-minute lecture per week for 10 weeks, in a theater-style lecture hall with 72 students, as well as one 3 -hour lab period. Classroom interaction during the lecture was close to nil. Our first change was to break this lecture for 72 students into three sections of 24 students each. With individual instructors 
managing smaller classes, instead of large team-taught lectures, we gain the benefits of smaller class size.

The resulting class schedule is organized as shown in Table 1, with three lectures on Monday followed by one lab period for each section throughout the week. In their class schedules, all students are assigned by the Registrar to all three hours on Mondays. Students attend only one of the three lectures, per a calendar provided in the syllabus. Lectures 1, 2 and 3 on any given Monday are identical. Depending on instructor preferences, one instructor can give all three lectures or the three instructors can each give their own.

Table 1: New schedule of lecture and lab periods

\begin{tabular}{|c|c|c|c|c|c|}
\hline Hour & M & $\mathrm{T}$ & $\mathrm{W}$ & $\mathrm{R}$ & $\mathrm{F}$ \\
\hline 1st & $\begin{array}{c}\text { Lecture } \\
\text { Section 1 }\end{array}$ & & & & \\
\cline { 1 - 1 } 2nd & $\begin{array}{c}\text { Lecture } \\
\text { Section 2 }\end{array}$ & $\begin{array}{c}\text { Lab period } \\
\text { Section 1 }\end{array}$ & \multicolumn{1}{|c|}{$\begin{array}{c}\text { Lab period } \\
\text { Section 2 }\end{array}$} & $\begin{array}{c}\text { Lab period } \\
\text { Section 3 }\end{array}$ \\
3rd & $\begin{array}{c}\text { Lecture } \\
\text { Section 3 }\end{array}$ & & & & \\
\hline
\end{tabular}

\section{Improvement no. 1: Turning lectures into labs}

There are 10 lecture periods in a term (one per week), currently comprising 4 lectures and 6 "mini-labs." The six mini-labs are the heart of the "turning lecture into labs" idea, but for completeness, we'll briefly discuss the other four lecture periods too.

In week 1, the lecture hour and lab period (a workshop) constitute a single 4-hour learning module covering uncertainty analysis. In lecture, students learn the principles they will apply in the workshop later that week. In week 2, the lecture/workshop pattern repeats, covering experiment design. In week 3 , the lecture period is devoted to technical credibility (calibration, uncertainty in calibration, outliers). In week 4, the lecture is replaced by an in-class activelearning exercise (sort of a mini-workshop) in applying the course standards for graphs, tables, and equations.

In weeks 5 through 10 , the six lectures have been replaced with six 50 -minute mini-labs. The topics covered by the mini-labs include the operating principles and performance characteristics of commonly-encountered measurement devices such as load cells, pressure transducers, and potentiometers - topics traditionally covered in a lecture format. Via a pre-lab exercise, students learn the operating principles of a specific measurement device. In the mini-lab, they connect the device (with little direction from the instructor) using their knowledge of the principles of operation and specifications or data provided by the manufacturer - a situation encountered by every experimentalist on first taking an instrument out of its box. The mini-lab reproduces this experience for the student.

Table 2 summarizes the six mini-labs. The "unscripted" activities are those for which students are given no explicit directions. For example, the transducer in the second mini-lab is a linear potentiometer. In the prelab, students make a preliminary estimate of the transducer's sensitivity 
from the manufacturer's specification sheet and practice a data reduction technique to determine the uncertainty in a slope. In the mini-lab, students perform an informal calibration of the transducer to confirm their estimate of sensitivity, but they are not told how to connect the wires of the transducer to the power supply and multimeter. Students use the potentiometer to measure displacement to determine a spring constant and uncertainty, but they are not told how to compute the total uncertainty associated with their measurement. (The full text of the potentiometer mini-lab and prelab are given in the Appendix.)

Table 2: Summary of the six mini-labs

\begin{tabular}{|l|l|l|}
\hline Transducer & Description & Unscripted activity (we don't tell them how) \\
\hline Load cell & $\begin{array}{l}\text { Informal calibration of a 5 lb load cell, } \\
\text { i.e., experimentally confirm its sensitivity. }\end{array}$ & $\begin{array}{l}\text { Making wiring connections. Interpreting } \\
\text { readouts. }\end{array}$ \\
\hline $\begin{array}{l}\text { Linear } \\
\text { potentiometer }\end{array}$ & $\begin{array}{l}\text { Informal calibration of a } 900 \mathrm{~mm} \text { "string- } \\
\text { pot." Experimentally find a spring } \\
\text { constant. }\end{array}$ & $\begin{array}{l}\text { Making wiring connections. Interpreting } \\
\text { readouts. Choosing appropriate elements of } \\
\text { the uncertainty analysis. }\end{array}$ \\
\hline $\begin{array}{l}\text { Pressure } \\
\text { transducers }\end{array}$ & $\begin{array}{l}\text { Informal calibration of a 1 psi strain gage } \\
\text { pressure transducer. }\end{array}$ & $\begin{array}{l}\text { Making wiring connections. Interpreting } \\
\text { readouts. Using a U-tube manometer as the } \\
\text { calibration standard. }\end{array}$ \\
\hline $\begin{array}{l}\text { Rotary digital } \\
\text { encoder }\end{array}$ & $\begin{array}{l}\text { Informal calibration. Interpreting } \\
\text { readouts. Estimating uncertainties. }\end{array}$ & $\begin{array}{l}\text { Deciphering manufacturer's DIP switch } \\
\text { settings. Making wiring connections. } \\
\text { Interpreting readouts. Determining the system } \\
\text { resolution. }\end{array}$ \\
\hline Tachometers & $\begin{array}{l}\text { Compare small-motor speeds measured } \\
\text { with two different tachometers. Repeat at at } \\
\text { a different motor speed. }\end{array}$ & $\begin{array}{l}\text { Deciphering manufacturer's DIP switch } \\
\text { settings. Making wiring connections. } \\
\text { Interpreting readouts. Exploring how to } \\
\text { increase transducer sensitivity. Determining } \\
\text { the system resolution. Choosing appropriate } \\
\text { elements of the uncertainty analysis. }\end{array}$ \\
\hline Accelerometer & Undetermined, still in development & Undetermined, still in development \\
\hline
\end{tabular}

With the mini-labs, we've "rewritten out the script" by 1) replacing a conventional lecture with a hands-on experience with improved individual accountability — we have enough mini-lab equipment for students to do this work in pairs; and 2) by giving students less-detailed instructions than we did in the past.

The learning objectives of the mini-labs can be summarized as follows. After completing the mini-labs, students should be able to: explain the principles of operation of various transducers; determine resolution, range and sensitivity of each measurement system encountered; interpret readings from the measurement system; and determine the uncertainties associated with an application of the measurement system. These objectives are typical of conventional measurements courses, here accomplished through active, hands-on experiences.

\section{Improvement no. 2: Replacing labs with workshops}

Prior to the changes we describe, the ten lab periods of the course were used for six fully-scripted experiments, three project periods, and one oral presentation period. The changes are:

- two of the experiments have been replaced with workshops, 
- one of the project periods has been rescheduled as an design exercise for the project, applying the principles learned in the workshops,

- the oral presentation period has been moved to finals week, giving students one additional lab period for project work.

The resulting lab schedule now has two workshops, four experiments, and four project periods (one of which is devoted to project design), with the oral presentation given during finals week in lieu of a final exam.

As previously stated, the lecture/workshop in week 1 constitutes a single 4-hour learning module covering uncertainty analysis. In the workshop, working individually, students are given a "guided tour" through an uncertainty analysis for an illustrative experiment. The workshop illustrates the common types of uncertainty students are likely to encounter during the term in their projects, labs, and mini-labs and serves as a ready reference for the rest of the term. Intermediate answers are provided in the text of the workshop handout so that students can check their work as they go. The workshop objectives are to: calculate systematic and random uncertainties; propagate uncertainties; calculate total uncertainty; and reduce uncertainty by modifying the measurement system or the measurement process.

In week 2, the combined lecture/workshop covers the use of uncertainty analysis to design an experiment, based on an approach described by Coleman and Steele [4]. In the workshop, again working individually and with intermediate answers given, students apply the concepts of uncertainty magnification factors (UMFs) and uncertainty percent contributions (UPCs) to the problem of designing a cantilever-beam experiment to determine the beam's elastic modulus. On completing this workshop, students should be able to perform general uncertainty analysis in the experiment design phase to determine the feasibility of a proposed experiment, to determine which measurements might be more important than others, to select experimental parameters and property values to meet realistic uncertainty bounds on the experimental result, and to suggest appropriate instrumentation.

In week 3, the lab period is devoted to the project. But rather than allowing students to begin fabrication and testing, we have substituted Workshop 3: Experiment Design in which the student teams are required to apply the concepts of Workshop 2 to the design of their particular term project. Students use their completed materials from Workshop 2 as a template for their design. In this design workshop, however, there is no "guided tour" nor any intermediate answers since students are exploring the parameters of their own experimental design project.

Recalling our four main categories of lab learning objectives-instrumentation, experiment, analysis, and learn from failure - Workshop 1 satisfies the analysis objective, Workshop 2 satisfies the experiment objectives, and Workshop 3 satisfies the instrumentation and experiment objectives. Two workshops planned for future implementation are data acquisition and calibration.

\section{Improvement no. 3: Constraining the measurements projects}

In the previous version of the course, students selected their own projects. "Muzzle velocity of a potato gun" is an example. They were often topics of interest to the students - a definite 
advantage - but the problems with time management as the students struggled to define the problem outweighed this advantage. By carefully selecting a menu of 6 projects that we know are feasible, we focused student attention on analyzing their data and presenting it in a credible manner, rather than on simply getting the data.

We recognize that it is beneficial to struggle with ill-defined, open-ended problems and find a way to get the data; however, the measurements project was not the right venue for this learning objective. Rather, we moved that open-ended project to the senior-level lab course, after the students had learned (a) how to confidently use several common transducers, and (b) how to collect, analyze, and present data in a credible manner for a well-defined problem.

In the week-4 lab period, devoted to the project, students are generally able to fabricate the apparatus they designed in week 3 and make a preliminary measurement. At this early stage, our objective is for students to get a rough value for their result even if they haven't had time to assemble all the transducers they plan to use. It is intended to give them an early start on getting the apparatus assembled, and verify the feasibility of their design.

Three of the six student project solicitations used in the Winter Quarter 2005-06 are listed in Table 3. (Three additional project solicitations are listed in Appendix 3.) The two basic instructions to all student teams are:

1. Objectives. The objective of your experimental design project is to make a measurement that your skeptical customers (your instructors) agree truly represents your measurand. Convince us of the technical quality of your results and communicate those results in an oral presentation that is coherent and well-rehearsed. We emphasize both technical quality and communications quality.

2. Solicitations. The measurements projects we'll be doing this quarter are described in the list of project solicitations. Teams will select and rank their three top choices. When assigning projects, we attempt to give every team one of their top three choices.

Table 3: Experimental projects in mechanical measurements

\section{No. 1: Friction Factor}

Goal. Determine the friction factor (D'Arcy friction factor) for water flow through a pipe. Your results should have a $95 \%$ confidence interval of $\pm 15 \%$ or better.

Some ideas to help you get started. The friction factor is associated with the pressure drop across a length of pipe. The basic principle describing this flow is the mechanical energy balance (modified Bernoulli's equation). The length and diameter of pipe, pipe material, and flow rate, are all up to you. Measurands may include flowrate, pressure drop, pipe length and diameter.

Caution. Water is messy! Equipment assembly may take time.

\section{No. 2: Mass Moment of Inertia}

Goal. Determine the mass moment of inertia of a bicycle wheel (or similar rotating body). Your results should have a $95 \%$ confidence interval of $\pm 10 \%$ or better. Also determine the mass moment of inertia using the appropriate theory and improve the theoretical model until the theoretically-predicted value lies on the experimental confidence interval. 
Some ideas to help you get started. This required approach is called a "bifilar pendulum" (a handout with the necessary theory is available). An object is suspended using two threads and is set to oscillate about a vertical axis. The mass moment of inertia is found from measurements of the frequency of rotation and system dimensions. Read your text for ideas on measuring the period of oscillation, mass and dimensions. Experiment parameters to be determined during the design phase include system dimensions and the number of trials to minimize uncertainty .

Caution. To achieve good results, the threads may have to be suspended from a high-bay ceiling. The object (e.g., the bicycle wheel) has to remain in planar orientation as it oscillates.

No. 3: Heat Transfer Coefficient

Goal. Determine the convective heat transfer coefficient $h$ for a electric resistive element exposed to air. Your results should have a $95 \%$ confidence interval of $\pm 20 \%$ or better.

Some ideas to help you get started. The basic principle involved is Newton's law of cooling for convective heat transfer. Either forced convection or natural convection may be examined. Measurands may include voltage, current, surface temperature, air temperature and surface area.

Caution. The reading from a thermocouple attached to a surface is not necessarily the true temperature of the surface.

From the descriptions given in the table, the reader should note that most decisions about the general approach to the problem are given to the students. By narrowing the focus of the projects in this way, we have them spend less time than formerly on brainstorming and other open-ended design tasks (these important skills are covered elsewhere in the curriculum in more than one course), and more time than formerly making credible experimental design choices based on uncertainty analysis and available measurement systems - precisely the main learning objectives of the course.

\section{Improvement no. 4: Rewriting the experiment script}

During the lab periods of the final six weeks of the term, student have two periods devoted to their projects and four periods devoted to larger-scale laboratory experiments. In the past, these experiments have been fairly well-scripted, that is, students would follow a detailed procedure, making no decisions about what data to record under what operating conditions, how many trials to make, what graphs to make to show the results, and so forth.

By "rewriting the script" for these labs, we put more responsibility on the students to make experimental decisions. The experiments have all necessary equipment assembled, but not necessarily connected. The transducers used are: Pitot-static tube, rotameter, turbine flowmeter, dead-weight pressure tester, Bourdon tube pressure gage, thermocouple, thermistor, dynamometer, and an optical tachometer. The four experiments can be roughly categorized as a temperature transient response experiment, a dynamometer experiment, a flow experiment, and a calibration experiment. We have built two copies of each experiment so that students work in pairs instead of in teams of four, increasing the likelihood that students stay engaged.

These larger-scale experiments are similar to the mini-labs in that the objective is to give students experience with transducers, but they differ in that the use of them is more time- 
consuming, and how to make the measurement is less well-defined. For example, in the flow experiment, the students are explicitly told to "play around" with the instruments, in order to develop confidence in their use. In the dynamometer experiment, the students are given a specific goal- "Develop an experiment to determine the torque-speed characteristics of the DC motor. Vary the load from no-load to locked-rotor conditions. Plot the resulting torque-speed curve with confidence intervals ("error bars"). Your uncertainty goal is an average relative uncertainty of $10 \%$ or less with $95 \%$ confidence. Stringently adhere to all safety requirements." - but with no procedural details. The students have to decide what data to take, how many trials, how to assess uncertainties, and so forth. This aspect of "rewriting the script" helps us avoid the "collect the data and get out" tendency inherent in our traditional labs.

\section{Results}

Recall that this paper focuses on four course improvements: 1) replacing lectures with mini-labs; 2) replacing labs with workshops; 3) narrowing the student project focus; and 4) "rewriting the script" of conventional experiments. The results enumerated below address these four topics.

1. (mini-labs) Student confidence with transducers in the follow-on senior laboratory course is improved. Since we've started giving them "hands-on" experiences hooking up and checking out transducers using instructions from the manufacturer (i.e., not step-by-step instructions from us), they are now less afraid to try hooking up new ones. Our technicians report that students are more likely to try to hook up a transducer, and ask for help if it doesn't work, rather than ask the technicians to set it up for them right from the start.

2. (workshops) A colleague teaching the follow-on senior laboratory course made the unsolicited comment that the quality of the uncertainty analyses is a notch better than before.

3. (workshops) The quality of the questions our students ask us is improved, especially in the context of experiment design. Approximately $30 \%$ of the groups ask questions about how their choices for their experimental apparatus will affect the uncertainty in their results. For example, in an orifice meter experiment, we have discussed how a smaller diameter pipe results in a higher pressure drop and may therefore result in a lower percentage uncertainty in pressure. On the other hand, it may also result in a higher uncertainty in the measurement of diameter. Prior to our including experiment design exercises in the course, these discussions rarely happened, even though the students had to make the same decisions about the particulars of their experimental apparatuses.

4. (narrow the project focus) Working prototypes of project experimental apparatuses are appearing after 4 to 5 weeks under the new organizational scheme compared to 6 to 8 weeks under past schemes.

5. (narrow the project focus) The oral presentations given at the end of the term are more credible. We no longer see results such as a drag force less than zero. We are in the process of collecting data over time in order to assess the improvement in technical credibility. The evaluations of these oral presentations are used in our ABET program outcomes documentation.

6. (rewriting the experiment script) Students were more actively engaged in using the lab equipment and are forced to communicate and struggle through the lab exercises. 
Previously, with four students per team and more scripted instructions, it was difficult for all four to gain meaningful experience using the "canned" experimental apparatuses.

7. (rewriting the experiment script) Students typically remain in the lab for the full 3 hours, sometimes completing and submitting the entire assignment before leaving, and are asking more questions about the experiment than in the past. Previously, it was customary for them to leave shortly after they had collected their data (the tendency to "collect the data and get out" [2]), and infrequently come see us for help afterwards.

We recognize these are not quantitative results, and make no claim to their universal applicability, but nonetheless we are encouraged by these subjective indicators.

\section{Conclusions}

Based on these subjective results, we draw the following conclusions regarding the four basic course improvements.

1. Minilabs using transducers are more engaging and more effective at helping students learn, compared to the lectures they replaced.

2. Workshops in uncertainty analysis and experiment design resulted in better application of uncertainty principles than the previous lecture plus homework approach.

3. Narrowing the focus of projects allowed students more time to analyze and make sense of their data and present more convincing results.

4. Fewer instructions and teams of 2 rather than 4 appear to have improved student engagement, making some headway in addressing the "take the data and get out" mentality.

Appendix 1: Prelab for the Potentiometer Mini-Lab (an example of the required preparation for a mini-lab)

\section{Instructions}

1. Individually complete the reading and the prelab questions before coming to class.

2. Develop your solution on engineering paper. Show all your work, as you would in a conventional homework problem.

3. Read section 8.2.1 (potentiometers) in the text and the principle of operation below.

4. Review the attached specification sheet for Celesco 632169 (Rev. P1) linear potentiometer.

5. Report results in US customary units.

Principle of operation. A thin, nylon-coated, stainless steel cable is wrapped around a drum inside the potentiometer. The loop at the end of the cable is attached to the item being displaced. As displacement increases the cable extends, turning the drum, causing the voltage divider output to vary. The displacement must not exceed the cable length. The drum on which the cable is wound is spring-loaded, so the cable stays in tension, applying a force to the system being measured.

Uncertainty in a slope. Often the slope of a linear regression is the quantity of interest rather than the regression equation. Given $\{x, y\}$ data, the slope $m$ is determined using the formula 


$$
m=\frac{N \sum_{i=1}^{N} x_{i} y_{i}-\sum_{i=1}^{N} x_{i} \sum_{i=1}^{N} y_{i}}{N \sum_{i=1}^{N}\left(x_{i}\right)^{2}-\left(\sum_{i=1}^{N} x_{i}\right)^{2}} .
$$

If we can assume that for all data $\left(x_{i}, y_{i}\right)$ the uncertainty in $x_{i}$ remains constant and the uncertainty in $y_{i}$ remains constant, that is,

$$
\begin{aligned}
& w_{x_{i}}=w_{x_{i+1}}=\text { constant } \\
& w_{y_{i}}=w_{y_{i+1}}=\text { constant }
\end{aligned},
$$

then it can be shown that the uncertainty in slope is given by (where $\bar{x}, \bar{y}$ are the sample means)

$$
w_{m}^{2}=\frac{\sum\left(y_{i}-\bar{y}\right)^{2}}{\left[\sum\left(x_{i}-\bar{x}\right)^{2}\right]^{2}} w_{x}^{2}+\frac{1}{\sum\left(x_{i}-\bar{x}\right)^{2}} w_{y}^{2} .
$$

Prelab questions (to be completed before coming to class)

1. State the potentiometer's measurand.

2. State the force applied by the cable to the system being measured.

3. Determine the system sensitivity.

4. For the data shown in the table, determine the slope of $y(x)$ and its uncertainty, i.e., find $m \pm w_{m}$. Assume $w_{x}=0$ in and $w_{y}=0.25 \mathrm{lbf}$.

\begin{tabular}{c|c}
\multicolumn{2}{c}{ Data Table (Ques. 4) } \\
\hline$y$ (lbf) & $x$ (in) \\
\hline 5 & 1.22 \\
10 & 2.31 \\
15 & 3.40 \\
20 & 4.50
\end{tabular}

Appendix 2: Potentiometer Mini-Lab (an example of the in-class mini-lab activities)

Instructions. This is a "hands-on" experience. Physically use the transducer (change the input, examine the output) to develop solutions to the following problems. Refer to the prelab, the textbook, and the manufacturer's documentation for assistance. Read! Ask questions!

- Each pair of students turns in one completed assignment.

- Develop your solution (not just answers) on engineering paper and show all your work, as you would in a conventional set of problems.

- Report all results in US units (lbf, in). A mass of $1 \mathrm{~kg}$ weighs $2.207 \mathrm{lbf}$.

- Mini-lab reports are due by 5 pm Tuesday (the day following the mini-lab).

Prelab. With your partner, review your solutions to the prelab questions. Agree on a solution.

Sensitivity and resolution. Remove the potentiometer from the storage box. Do not apply power until you are certain the wiring is correct.

5. Take 3 or 4 readings using the aluminum meter stick as the known reference input. Sketch a calibration curve and estimate the potentiometer sensitivity (you can use a computer package if you like). Compare your experimental result to the expected transducer sensitivity.

6. Determine the resolution of the measurement system. Explain your approach.

Application. Hang the spring from the eyebolt. Use the weights as reference force inputs (you can safely hang up to $2 \mathrm{~kg}$ ). 
Holding the potentiometer on top of the beam, pull down on the cable (without bending the cable) to measure spring deflection.

Record data to determine the spring constant. A spring curve is conventionally plotted as $F(x)$, where the slope of the curve is the spring-constant $k$, and the uncertainty in the slope is $w_{k}$.

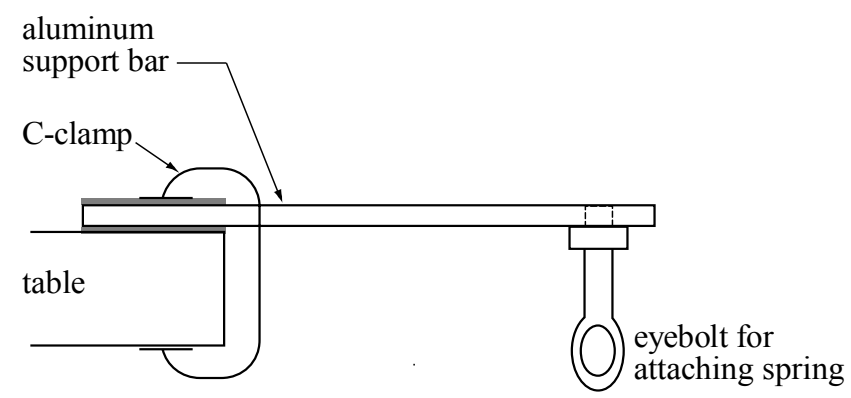

Fig. 1: Fixture for determining a spring constant.

Put away the hardware. Carefully disconnect everything you assembled. Return everything to the storage box, including the manufacturer's documentation. Turn off instrument power.

Analysis. From prior experiments in which we calculated the uncertainty in this spring constant, we have determined that:

- the $w_{x}$-term (displacement) is negligible compared to the $w_{y}$-term (applied force).

- for our standard weights larger than $200 \mathrm{~g}$, the uncertainty in weight is $\pm 0.00025 \mathrm{lbf}$.

7. Determine your spring constant $k \pm w_{k}$. Show all your work.

8. Assume you are given a seemingly identical spring from the same manufactured batch. Assume that the experiment you just performed has been repeated using 30 springs from that batch, producing a sample standard deviation in the spring constant of $S_{k}=0.025$ $\mathrm{lb} /$ in. Determine a new estimate of $k \pm w_{k}$ for the batch. Show all your work.

Finishing up. Use the cover sheet provided and assemble your complete solution in the order shown on the cover sheet.

Appendix 3: Additional project solicitations (illustrating the projects' focused scopes of work)

\section{No. 4: Force-Deflection behavior of a Compliant Device}

Goal. Determine the force-deflection characteristics of a compliant (spring-like) device. Your results should have a $95 \%$ confidence interval of $\pm 5 \%$ or better.

Some ideas to help you get started. If you select this project, your team must propose three different systems as possible force-deflection systems to be measured (examples of compliant devices are skis, bungee cords, a torsion rod, and a compliant surgical device). You will probably measure applied forces and the resulting displacements. Likely requirements include multiple measurements of load and displacement over the entire elastic range of the spring, linear regression, and testing of outliers. Parameters to be determined during the experiment-design phase include the range of forces and the number of trials. 
Caution. While the data reduction seems simple, the analysis is more challenging that it appears at first glance.

\section{No. 5: Flow Meter Discharge Coefficient}

Goal. Determine the discharge coefficient for an orifice flow meter. Your results should have a $95 \%$ confidence interval of $\pm 10 \%$ or better. Use water as the working fluid.

Some ideas to help you get started. You will need to construct a simple orifice flow meter. PVC pipe is a good, inexpensive choice for material. The text describes these flowmeters nicely. Parameters to be determined during the experiment-design phase include flow rate, pipe diameter, orifice diameter, and the number of trials.

Measurands may include flow rate, pressure difference, pipe diameter, and orifice diameter.

Caution. Water is messy! Assembling the apparatus may take time.

\section{No. 6: Wind Tunnel Turbulence Measurement (Drag On A Sphere)}

Goal. Determine the turbulence factor of the RHIT open-circuit wind tunnel. Your results should have a 95\% confidence interval of $\pm 20 \%$ or better.

Some ideas to help you get started. The turbulence factor $T F$ is defined as follows:

$$
T F=\frac{385,000}{\operatorname{Re} \text { at which } C_{D, \text { sphere }}=0.30}
$$

In order to find TF you will need to measure drag on a sphere at several Reynolds numbers. The drag coefficient should vary with Reynolds number; you must determine the Reynolds number which results in a drag coefficient of 0.30 . Equipment you might use includes the wind tunnel and force balance, equipment to measure dimensions of the sphere, and equipment to measure air temperature and pressure. Parameters to be determined during the experiment-design phase include the diameter of the sphere, the air speeds to use, and the number of trials.

Caution. Plan to spend some time acquiring familiarity with the wind tunnel, the balance, and the manometer.

\section{References}

[1] Layton, R.A., Mech, A.R. and Mayhew, J.E. (2004), Ideas into action: Using learning objectives to revitalize a mechanical engineering laboratory sequence, in proc. ASME Intl Mech. Engng Congr \& Expo., Dyn. Sys. \& Control Div., Anaheim.

[2] Sanders, L.K. (2002) A novel structure for measurement and instrumentation courses, in proc. ASME Intl Mech. Engng Congr \& Expo., Dyn. Sys. \& Control Div., New Orleans.

[3] Feisel, L.D. and Peterson, G.D. (2002) A colloquy on learning objectives for engineering education laboratories, in proc. ASEE Annual Conference, Montreal.

[4] Coleman, H.W. and Steele, W.G. (1999) Experimentation and Uncertainty Analysis for Engineers, 2/e, Wiley. 